\title{
Static Analysis and Improvement of Ranging Arm of Shearer Based on ANSYS
}

\author{
Lirong Wan ${ }^{1,}$, Zehua Wang ${ }^{1, b^{*}}$, Zhaosheng Meng ${ }^{1, c}$,Yawei Zong ${ }^{1, d}$ \\ ${ }^{1}$ College of Mechanical and Electronic Engineering, Shandong University of Science and \\ Technology, Qingdao 266590, China \\ awanlr666@163.com, 'bangzehua910515@163.com,,1607999059@qq.com, \\ d591656986@qq.com
}

Key words: the shell of the shearer ranging arm; force analysis of the drum; static analysis; the improvement of the shell

\begin{abstract}
In order to optimize the shell structure of the shearer ranging arm, the force of the shearer drum is analyzed, and it used three-dimensional software Solidworks to establish the shell entity model. The structural static analysis of the shell is implemented by ANSYS software. Then it attained the static stress and deformed state of the shell. According to analysis results, the design of the shell structure was optimized.It concluded that the maximum deformation of the ranging arm is at the drum shaft, and it also corresponds to the maximum stress, and mechanical properties and the reliability of the shell were significantly improved.
\end{abstract}

\section{Introduction}

The shell of the ranging arm is one of the main parts of shearer cutting unit ${ }^{[1]}$, design and manufacturing quality of its structure have a great impact on cutting unit's load distribution , carrying capacity, etc. Shearer work process by the cutting resistance, traction resistance, loading coal reaction force, axial force and so on, eventually passed to the ranging arm, when these forces are relatively large, ranging arm is easy to damage or deformation too large. This requires us to make finite element analysis of ranging arm, and obtained ranging arm's stress and deformation state, on this basis, it improve the ranging arm, thereby improving the quality of the ranging arm.

\section{shearer drum's stress analysis}

During the operate of the shearer, the force of the drum is very complex, and therefore, the forces need to be simplified. So we can assume that the drag on the drum can be decomposed into cutting resistance and traction resistance and axial forces, and are concentrated on the center of the drum. $^{[2]}$

Below is the force diagrams of the drum(Figure 1).

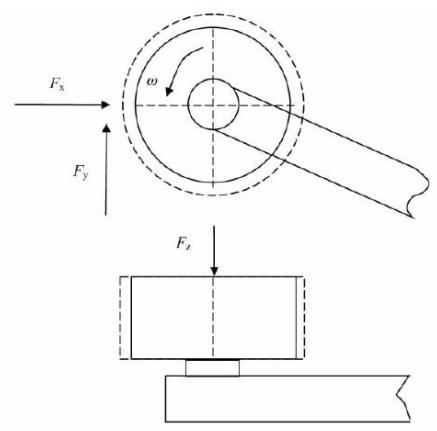

Fig. 1 force diagrams of the drum 
Under the conditions of shearer parameters have been identified, according to shearer cutting power to estimate the force of the drum. ${ }^{[3,4]}$

$$
\begin{aligned}
& F_{x}=K \frac{1.91 \times 10^{7} N \eta}{n D} \\
& F_{y}=\frac{T(47 \sim 59) \%}{1+K_{1}} \\
& F_{z}=0.2 F_{x}
\end{aligned}
$$

$F_{x}$ is simplify centralized cutting resistance, $\mathrm{N}$;

$F_{\mathrm{y}}$ is simplify centralized promoting resistance, $\mathrm{N}$;

$F_{\mathrm{z}}$ is axial force suffered by drum, N;

$K$ is cutting resistance and shear strength of the circumferential scale factor, Generally take $\mathrm{K}$ $=0.8$

$N$ is cutting unit motor's rated power, $\mathrm{kW}$;

$\eta$ is cutting unit mechanical transmission efficiency;

$n$ is drum Speed, $\mathrm{r} / \mathrm{min}$;

$D$ is drum diameter, $\mathrm{mm}$;

$T$ is the maximum traction of the shearer, $\mathrm{N}$;

$K_{1}$ is the ratio of cutting resistance of the front and rear drum, take $K_{1}=0.2-0.8$

MG650/1620 Shearer relevant parameters: Cutting power $\mathrm{N}=650 \mathrm{~kW}$; Drum Speed $\mathrm{n}=25.83 \mathrm{r} / \mathrm{min}$; Drum diameter $\mathrm{D}=2000 \mathrm{~mm}$; Cutting unit mechanical transmission efficiency $\eta=0.8599$; Traction $\mathrm{T}=775 \mathrm{kN}$.

Thus can be drawn:

$$
\begin{aligned}
& F_{x}=1.6468 \times 10^{5} N \\
& F_{y}=2.4219 \times 10^{5} N \\
& F_{z}=3.2936 \times 10^{4} N
\end{aligned}
$$

\section{Establish finite element model}

\section{Geometric model}

Using three-dimensional design software Solidworks ${ }^{[5]}$, according to MG650 / 1620 Shearer Rocker design dimensions, omitting irrelevant Chamfering, holes and so on ${ }^{[6]}$, and establish the three-dimensional solid model of the shell of shearer ranging arm (Fig. 2).

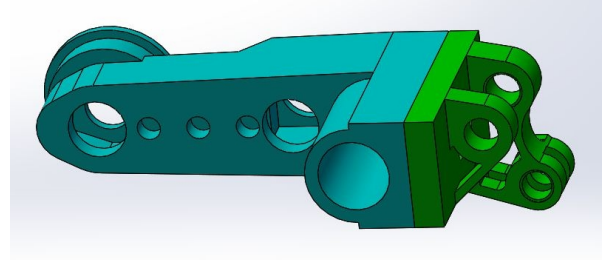

Fig. 2 Three-dimensional model of the shell of the ranging arm 


\section{Choose unit}

Solid element's shape may be tetrahedral or hexahedral, shape options with different grid types is different, generally tetrahedral element mesh for freedom, hexahedral elements for mapped meshing. The analysis unit chosen ANSYS tetrahedral unit 187 provided, SOLID187 is high-end three-dimensional 10-node solid element, has a secondary displacement, adapted to generate irregular meshes, and each node has three degrees of freedom: the nodal coordinate system's translation of $\mathrm{x}, \mathrm{y}, \mathrm{z}$ directions.

\section{Meshing}

You can choose to use free mesh or grid mapping to divide the model to generate a finite element model. Free meshing of the solid model without any special requirements, even if the model is irregular also can be meshed.Mapped meshing requires face or body has regular shape, that certain criteria must be met. ${ }^{[7]}$ Since the ranging arm complex structure, using mapped meshing method is relatively difficult, this analysis uses free meshing methods, and using Smart Size control and local mesh size control to control the density of the grid to mesh ranging arm. Division results shown in Figure 3.

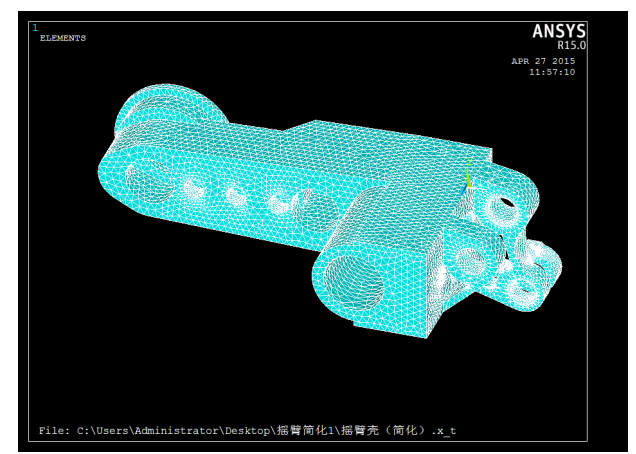

Fig. 3 Ranging arm meshing

\section{Applied load and boundary conditions}

\section{Equivalent load}

On the basis of shearer drum stress analysis, put the force equivalent to the ranging arm. And the force equivalent to the ranging arm obtain the following force and couple.

$$
\begin{aligned}
& R_{\mathrm{x}}=F_{x} ; R_{y}=F_{y} ; R_{z}=F_{z} \\
& M_{x}=R_{x} L_{a} ; M_{y}=R_{y} L_{b} ; M_{z}=R_{y} L_{c} \\
& L_{a}, L_{b}, L_{c} \text { is } R_{\mathrm{x}} 、 R_{\mathrm{y}} 、 R_{z} \text { arm, when the force point equivalent to the ranging arm . }
\end{aligned}
$$

The MG650 / 1620 parameter shows: $L_{a}=800 \mathrm{~mm}, L_{b}=800 \mathrm{~mm}, L_{c}=0 \mathrm{~mm}$, Thus

$$
\begin{aligned}
& R_{\mathrm{x}}=F_{x}=1.6468 \times 10^{5} \mathrm{~N} ; \\
& R_{y}=F_{y}=2.4219 \times 10^{5} \mathrm{~N} ; \\
& R_{z}=F_{z}=3.2936 \times 10^{4} \mathrm{~N} ; \\
& M_{x}=R_{x} L_{a}=1.93752 \times 10^{8} \mathrm{~N} \cdot \mathrm{mm} ; \\
& M_{y}=R_{y} L_{b}=1.31744 \times 10^{8} \mathrm{~N} \cdot \mathrm{mm} ; \\
& M_{z}=R_{y} L_{c}=0 \mathrm{~N} \cdot \mathrm{mm} ;
\end{aligned}
$$




\section{Applied load}

In order to facilitate loading, need to establish a node and assign a quality, unit MASS21 for this node, then directly set up a rigid body between the nodes and the cylindrical surface, so it can applied the equivalent of forces and moments to the node. Load is applied as shown in Figure 4

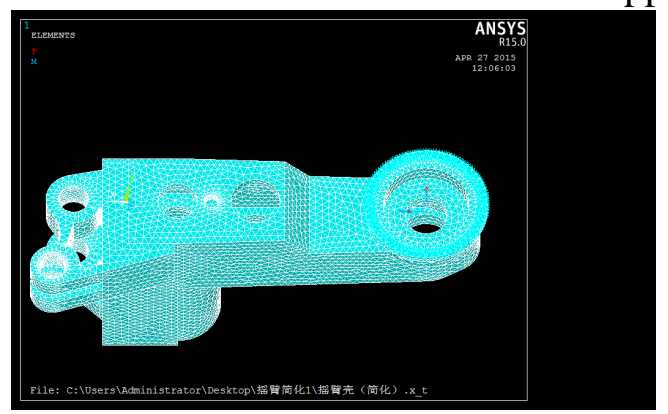

Fig. 4 Applied load

\section{Constraints imposed}

During the operation of the shearer, the drum in a different position has different stress state of the ranging arm. When the ranging arm with the horizontal maximum angle, the ranging arm has maximum stress. ${ }^{[8]}$ we can constraint 4 lug at all of its degrees of freedom, and make static analysis in the position of maximum stress of the ranging arm. Constraints imposed shown in Figure 5.

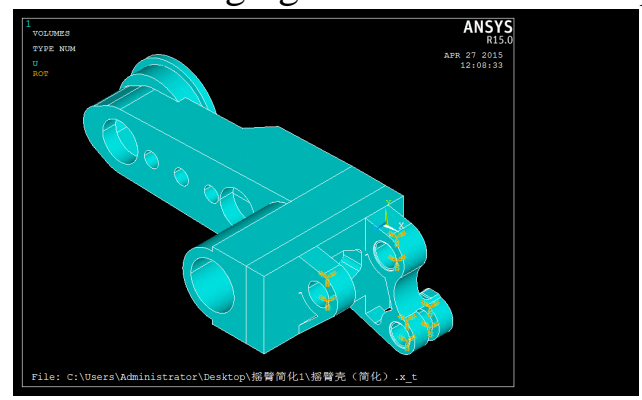

Fig. 5 Impose constraints

\section{Analysis result}

Though static analysis of the ranging arm, we can obtain the stress distribution and the deformation maps of displacement.(Figure 6)

As can be seen from the figure, the maximum stress at the ranging arm drum shaft, corresponding to the maximum deformation amount. Stress amplitude at ranging arm transition junction is also large, but less than the material allowable stress, meet the requirements of safe work.

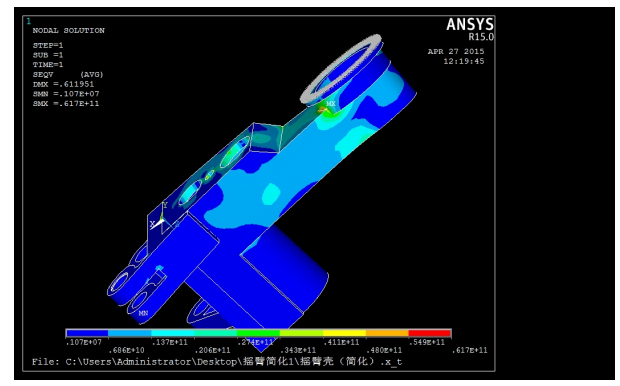

a.Stress distribution

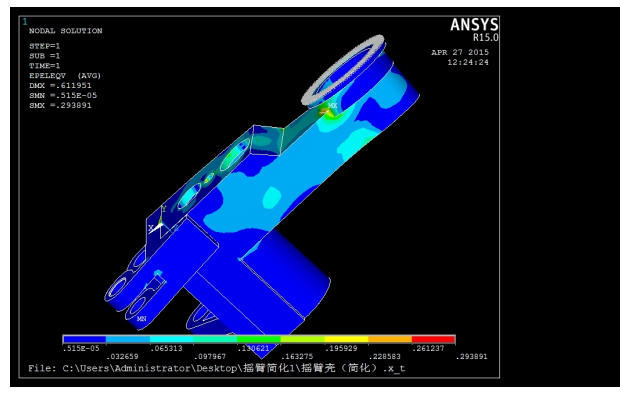

b.Displacement deformation maps

Fig. 6 Stress-strain diagram 


\section{Structure Improvement}

In order to reduce the amount of stress and deformation at the ranging arm drum shaft, add the ribs in the position shown in Figure 7.

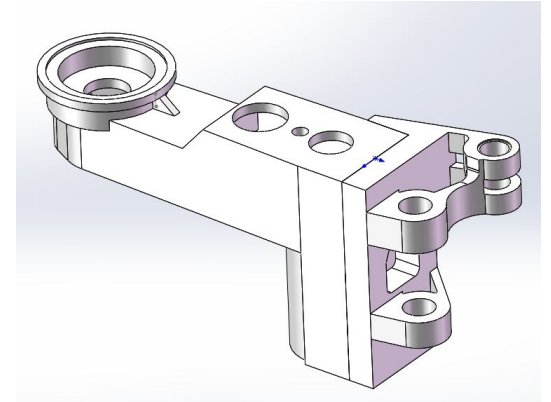

Fig. 7 Add ribs

After add the ribs, make finite element analysis again, it can obtain the stress distribution and the deformation maps of displacement(Figure 8).

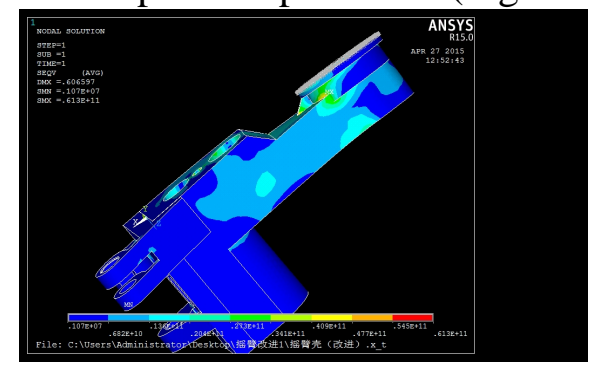

a.Stress distribution

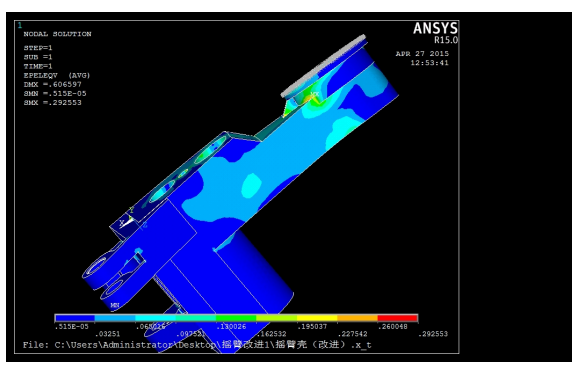

b.Displacement deformation maps

Fig. 8 Stress-strain diagram after structural improvements

The amount of stress change:

$$
\Delta_{1}=\frac{0.617 \times 10^{11}-0.613 \times 10^{11}}{0.617 \times 10^{11}}=6.483 \times 10^{-3}
$$

The amount of strain change:

$$
\Delta_{2}=\frac{0.293891-0.292553}{0.293891}=4.553 \times 10^{-3}
$$

Stress and strain can be seen clearly at ranging arm drum shaft becomes small.

\section{Summary}

In this paper, make structure static analysis by the finite element method on shearer ranging arm, from the analysis results, the maximum stress and deformation at the ranging arm drum shaft, where after add the ribs, stress and strain there is significant decreases.

In addition, the stress amplitude is larger at ranging arm transition junction, because the ranging arm transition junction is in alternating stress state, so it should be appropriate to increase the size of the transition region of the ranging arm, in order to avoid damage because of excessive stress.

\section{Acknowledgments}

The corresponding author of this paper is Zehua Wang. This paper is supported by National Natural Science Foundation of China (Grant No.51375282), Program for Changjiang Scholars and Innovative Research Team in University (Grant No.IRT1266), Innovative Research Team in 
University (Grant No.IRT1266), and Special funds for cultivation of Taishan Scholars and Special Program for independent innovation of Shandong Province (Grant No. 2013CXB40203). The authors have declared that no conflict of interest exists.

\section{References:}

[1] Xie Bo. Fatigue analysis on ranging arm of shearer in thin coal seam based on co-simulation of ANSYS and MSC Adams[J]. Computer Aided Engineering,2009, 18(2):34-38.

[2] Liu Chunsheng. Drum shearer theoretical design basis[M]. Xu Zhou: China Mining University Press, 2003.

[3] Wang Xiang. Rocker arm FEA preprocessing technology of shearer[J]. Mining Machinery,2009, 37(5): 8-11.

[4] Wu Weidong. Finite Element Analysis of Shearer Cutting Based on ANSYS[J]. Coal Mine Machinery,2009, 30(3):77-79.

[5] Zhan Yougang. Solidworks2012 Collection[M]. Bei Jing: Machinery Industry Press, 2012.8.

[6] Wu Yan. The CAE Analysis of Domestic Large Power Ranging Arm of Shearer[J]. Mine Electromechanical,2003,(5), 105-108.

[7] Song Zhian. Finite element analysis of mechanical structures[M]. Bei Jing: National Defense Industry Press, 2010.6.

[8] Luo Yanzhong. Finite element analysis of the shell of a shearer rocker[J]. Gui Hang academic research, 1998,3(4):36-40. 